\title{
THE TWENTY-SEVENTH ANNUAL MEETING OF THE AMERICAN MATHEMATICAL SOCIETY.
}

The twenty-seventh annual meeting of the Society was held at Columbia University on Tuesday and Wednesday, December 28-29, 1920, extending through two sessions on each day. The attendance included the following eighty-six members:

Professor J. W. Alexander, Professor R. C. Archibald, Professor R. A. Arms, Professor Clara L. Bacon, Dr. Charlotte C. Barnum, Professor W. J. Berry, Mr. William Betz, Professor G. D. Birkhoff, Professor Joseph Bowden, Professor R. W. Burgess, Professor B. H. Camp, Professor W. B. Carver, Professor F. N. Cole, Dr. G. M. Conwell, Professor J. L. Coolidge, Dr. W. L. Crum, Professor C. H. Currier, Dr. Tobias Dantzig, Professor J. V. De Porte, Dr. Jesse Douglas, Professor C. A. Fischer, Professor W. B. Fite, Professor C. H. Forsyth, Professor Tomlinson Fort, Mr. Philip Franklin, Dr. T. C. Fry, Professor R. E. Gilman, Professor O. E. Glenn, Professor W. C. Graustein, Dr. T. H. Gronwall, Professor Olive C. Hazlett, Professor E. R. Hedrick, Dr. A. A. Himwich, Professor L. A. Howland, Professor W. A. Hurwitz, Mr. S. A. Joffe, Professor Edward Kasner, Dr. K. W. Lamson, Mr. Harry Langman, Professor Gillie A. Larew, Professor Florence P. Lewis, Professor P. H. Linehan, Professor Joseph Lipka, Mr. L. L. Locke, Professor W. R. Longley, Professor C. R. MacInnes, Professor H. F. MacNeish, Professor R. M. Mathews, Professor H. H. Mitchell, Professor Frank Morley, Dr. H. M. Morse, Professor G. W. Mullins, Professor G. D. Olds, Professor F. W. Owens, Dr. Helen B. Owens, Mr. George Paaswell, Dr. Alexander Pell, Professor Anna J. Pell, Dr. G. A. Pfeiffer, Dr. E. L. Post, Professor H. W. Reddick, Professor R. G. D. Richardson, Dr. J. F. Ritt, Dr. G. M. Robison, Dr. J. E. Rowe, Professor F. H. Safford, Dr. Caroline E. Seely, Professor L. P. Siceloff, Professor Clara E. Smith, Professor P. F. Smith, Professor W. M. Smith, Professor Elijah Swift, Dr. J. S. Taylor, Professor H. D. Thompson, Mr. H. S. Vandiver, Professor Oswald Veblen, Professor J. N. Vedder, Professor J. H. M. Wedderburn, Professor H. S. White, Professor E. E. Whitford, Dr. Norbert Wiener, Professor A. H. Wilson, Professor Ruth G. Wood, Dr. T. S. Yang, Professor J. W. Young, Dr. S. D. Zeldin. 
President Frank Morley occupied the chair, relieved by Professors G. D. Birkhoff and R. G. D. Richardson, on Tuesday; Professors Richardson and H. S. White presided at the sessions on Wednesday. The Council announced the election of the following persons to membership in the Society: Professor L. M. Coffin, Coe College; Professor I. H. Fenn, Polytechnic Institute of Brooklyn; Dr. Ludwik Silberstein, Eastman Kodak Company; Dr. W. L. G. Williams, Cornell University. One hundred twenty-one applications for membership were received.

A report was received by the Council from Professor E. R. Hedrick, chairman of the committee on increase of membership and sales of publications, presenting one hundred ten applications for membership in the Society and sixty-six subscriptions to the Transactions. The report was accepted, with the thanks of the Council.

Professor T. S. Fiske, as representative of the contributors to the Bôcher memorial fund, tendered the fund to the Society to be held in trust and the income to be employed for the advancement of mathematical science. The trust was accepted, and a committee appointed to consider the most appropriate use to which the income of the fund could be devoted.

A committee was also appointed to make the necessary arrangements for the meeting of the Society to be held at Wellesley College in the summer of 1921.

The total membership of the Society is now 770, including 87 life members. The total attendance of members at all meetings, including sectional meetings, during the past year was 517 ; the number of papers read was 215 . The number of members attending at least one meeting during the year was 306 . At the annual election 189 votes were cast.

The Treasurer's report shows a balance of $\$ 8,994.53$, including the Life Membership Fund, which amounts to $\$ 7,518.87$. Sales of the Society's publications during the year amounted to $\$ 2,067.74$.

The Library now contains 5,862 volumes, excluding some 500 unbound dissertations.

The afternoon session on Tuesday was especially marked by the retiring presidential address of Professor Frank Morley, on Pleasant questions and wonderful effects. A dinner was held at the Faculty Club Tuesday evening, at which fifty members were present. 
At the annual election, which closed on Wednesday morning, the following officers and other members of the Council were chosen:

\author{
President, Professor G. A. Buiss. \\ Vice-Presidents, Professor F. N. Cole, \\ Professor Dunham Jackson. \\ Secretary, Professor R. G. D. Richardson. \\ Treasurer, Professor W. B. Fite. \\ Librarian, Professor R. C. ARChibald. \\ Committee of Publication, \\ Professor E. R. HEDRICK, \\ Professor W. A. HuRwitz, \\ Professor J. W. Young.
}

Members of the Council to serve until December, 1923,

Dr. T. H. Gronwall, Professor Florence P. Lewis, Professor O. D. Kellogg, Professor A. D. Pitcher.

At the close of the morning session on Wednesday, Professor H. S. White in a short address tendered the thanks of the Society to Professor Cole for his distinguished services during his long term of office as secretary of the Society and editor of the Bulletin. Professor White said in part:

"With the close of the year 1920, the resignation of Professor Frank Nelson Cole ends his long period of service as secretary of the American Mathematical Society. Since 1895 he has held that office, and since 1897 has been editor of the Society's Bulletin, a periodical issued ten times a year, and has published all the Society's programs and announcements. From these onerous duties, and others, he now withdraws.

"With deep regret we acquiesce. But we desire to express to our retiring secretary our gratitude for his labors. He has dignified and amplified his office. With few precedents to guide him, he has met with energy and tact its problems. The meetings of the Society he has prepared and managed with skill and eminent success. Its membership he has aided greatly to enlarge, maintaining cordial and helpful relations with all. As editor he has made the Bulletin increasingly interesting and useful, securing an expanding line of active contributors. He has maintained its high standards, while extending its range to keep pace with the augmenting activities of the Society.

"Surely fortunate is he who is able to sustain for twenty- 
five vears labors so varied and so strenuous; who can through steady, cumulative efforts develop and execute so fully his plans and policies. We extend to him our congratulations, in grateful recognition of his achievements; and we felicitate him now on his return to the engrossing problems of pure science."

The list of papers read at the meeting, together with abstracts of the papers, is as follows:

(1) Professor C. E. Wilder: Einstein's four-dimensional space is not contained in a five-dimensional linear space.

In 1880 Voss gave necessary and sufficient conditions that any curved manifold be contained in any higher-dimensional manifold. Using these conditions, Professor Wilder shows that any four-dimensional manifold that is contained in a fivedimensional linear space cannot satisfy the defining equations for an Einstein space.

(2) Dr. J. L. Walsh: On the convergence of the SturmLiouville series.

Dr. Walsh considers the differential system

$$
\begin{aligned}
u^{\prime \prime}(x)+\left[\rho^{2}-g(x)\right] u(x)=0 \\
(0 \leqq x \leqq 1, u(0)=u(1)=0),
\end{aligned}
$$

a special case of which is the differential system

$$
u^{\prime \prime}(x)+\rho^{2} u(x)=0 \quad(0 \leqq x \leqq 1, u(0)=u(1)=0),
$$

whose normal solutions are the functions $\{\sin n \pi x\}$. If $f(x)$ is an arbitrary function integrable and with an integrable square, there is formed the series composed of the term-byterm difference of the formal expansions of $f(x)$ in terms of the normal solutions of (1) and of (2); it is proved that this series converges uniformly and absolutely to zero on the entire interval considered. The method of proof is entirely elementary.

(3) Miss Anna M. Mullikin: Certain theorems concerning connected point sets.

Miss Mullikin establishes the following theorems.

I. If in a plane $S, m_{1}, m_{2}, m_{3}, \cdots$ is a countable collection of closed, mutually exclusive point sets, no one of which disconnects $S$, and if $m$, the sum of these point sets, is closed, then $m$ does not disconnect $S$. 
II. If in a plane $S, P$ and $Q$ are two closed point sets with no point in common and $H$ is a closed, bounded, connected set of points containing at least one point in common with $P$ and at least one point in common with $Q$, then $H$ contains a connected subset $H_{1}$, such that (1) $H_{1}$ contains no point in common with $P$ or with $Q$, and (2) each of the point sets $P$ and $Q$ contains at least one limit point of $H_{1}$.

(4) Dr. A. R. Schweitzer: On homogeneous functions as generators of an abstract field.

Dr. Schweitzer points out that the algebra of logic, the theory of abstract groups, and the theory of abstract fields, as special disciplines on iterative compositions, so far as is known, have been first emphasized by him. In further development of the abstract field as an iterative theory, he considers the possibility of generating an abstract field by rational functions of $m$ variables $(m \geqq 2),(1)$ homogeneous and integral of degree $\geqq 2,(2)$ homogeneous and rational of degree zero. In particular, he finds that the following functions may serve as undefined relations for an abstract field: $x^{2}-y z,(x+y) \cdot z,(x \pm y) \cdot y$, and $x / y-1$, the latter function being also quasi-transitive. In connection with the latter functions, it is worthy of note that the non-homogeneous functions $(1 \pm x) \cdot y$ generate a field under suitable postulational assumptions.

(5) Dr. A. R. Schweitzer: The concept of an iterative compositional algebra.

Dr. Schweitzer defines an iterative compositional algebra as a set $E$ of elements $a_{1}, a_{2}, \cdots, b_{1}, b_{2}, \cdots$, etc., subject to the following assumptions. Let $\lambda_{i}\left(a_{j} b_{j}\right)(i=1,2, \cdots, m$; $j=1,2, \cdots, n)$ be generating relations of a given (formal or material, proper or improper) pseudo-group $P G$ with a set (which may be vacuous) of adjoined conditions $R$ on the functions $\lambda_{i}$; then the author defines

$$
\Lambda_{i}(A B)=\left[\lambda_{i}\left(a_{1} b_{1}\right) \cdots \lambda_{i}\left(a_{n} b_{n}\right)\right]
$$

where $A$ and $B$ are $n$-ads in the sense defined by him in the American Journal of Mathematics (1909). Let the $n$-ads $\{A\}$ be elements of compositions $r_{s}\left[A_{1}, A_{2}, \cdots, A_{p}\right], p \geqq 2$, $s=1,2,3, \cdots$, where the $r_{s}$ satisfy a given set of equations in iterative compositions in the sense previously defined by 
the author. In particular, closure under the compositions $r$ may exist. The relation of the compositions $\lambda_{i}$ to $r_{s}$ will be the subject of special inquiry; in general, the $\lambda_{i}$ and $r_{s}$ satisfy explicitly stated equations in iterative compositions of order $\leqq i+s$. More generally, for the pseudo-group $P G$, one might substitute any set of necessary properties of any postulationally defined calculus of iterative compositions. See, e.g., Schroeder, Archiv (2), vol. 5.

(6) Professor Joseph Lipka: Transformations of trajectories on a surface.

In a paper read at the October meeting of the Society, Professor Lipka proved five geometric properties which completely characterize the trajectories on a surface for any positional field of force. In the present paper, a study is made of the point transformations which convert systems of curves on a surface $S$ possessing some or all of these properties into like systems on the transformed surface $S_{1}$. The general results are that systems of curves possessing the first property or the first two properties are invariant under an arbitrary point transformation, while all other systems are only invariant under a geodesic transformation. A similar study is made for the point transformations of " $n$ "-systems-brachistochrones, catenaries, velocity curves, etc.-on a surface.

(7) Mr. Harry Langman: Conformal transformations of period $n$ and groups generated by them.

Professor Kasner has studied the groups of transformations generated by conformal transformations of period 2 .

Mr. Langman's paper is principally concerned with a generalization of a problem studied by Kasner. The generalized problem, following Kasner's notation, consists in finding whether two transformations $f(z)$ and $g(z)$ can always be found such that $f_{m}(z)=g_{n}(z)=z$ and $g[f(z)]=F(z)$ identically for some integral values of $m$ and $n$. The subscripts here denote the iterated functions of corresponding order. In the more general problem no such numerical relation as that found by Kasner for the case $m=n=2$ is found necessary (other than $K_{1}{ }^{r}=1$ ). The complete result states that if $K_{1} \neq 1$, then $F(z)$ is always factorable; if $K_{1}=1$, the periods of the factor transformations, if they exist, must be equal; if $K_{1}=1$ and $K_{2} \neq 0$, then $F(z)$ is always factorable into two trans- 
formations of arbitrary period $>2$; if $K_{1}=1, K_{2}=K_{3}$ $=\cdots=K_{r}=0$, and $K_{r+1} \neq 0$, then $F(z)$ can not be factored into transformations of order $r$, or any factor of $r$, but can always be factored into transformations of any other order $>2$.

In the first part of the paper it is shown that if the transformation $F(z)$ is of period $n$, then it may be put uniquely into implicit form. The well known fact that $F(z)$ is conformally equivalent to a rotation about the origin is then readily deduced. The method also gives complete solutions of similar functional equations of the symbolic forms: $f^{m}=g$, where $g$ is given and $g^{n}=1 ; 1+f+f^{2}+\cdots+f^{n-1}=0$; etc. It is also shown that, corresponding to every function $f$, where $f^{n}=1$, there are others, $F$, satisfying the last functional equation.

Kasner has considered other transformations of period 2, termed by him conformal symmetries, defined by $f\left(z_{0}\right)$, where $z_{0}$ is the conjugate of $z$. It is here shown that no other kind of transformation of this type exists, i.e. that all reverse conformal transformations are of period 2 .

(8) Professor O. E. Glenn: On a new treatment of theorems of finiteness. Second paper. (Preliminary report.)

Professor Glenn's paper consists, in its present form, of two parts. A third part, dealing with certain amplifications, is to be added. The first part comprises a proof of the finiteness of binary formal modular concomitant systems (modulo $p$ ), which has been an outstanding unsolved problem since the publication of Dickson's first paper on modular invariants (1907) and of a paper by A. Hurwitz (1903). This proof is based upon the algorism on modular concomitants appertaining to domains mentioned in the present author's memoir in the Transactions for 1920 . The second part of the paper gives statements and solution of certain finiteness propositions in the realm of differential invariants. The present paper is, in regard to general method, a sequel to the one published under the same title in the Transactions for 1919.

(9) Professor J. E. Rowe: The efficiency of projectile and gun.

The purpose of this investigation is to formulate mathematically a means by which the efficiencies of different pro- 
jectiles fired in different guns may be compared, using as a criterion the energy wasted in propelling the projectile to the point of fall. It is possible also to obtain from this the economic efficiency, by taking into account the cost of the material used.

(10) Dr. S. D. Zeldin: On the structure of finite continuous groups with one two-parameter subgroup.

This paper considers groups with one two-parameter subgroup. By imposing certain conditions on groups meroedrically isomorphic with the given ones, the author shows how the structure can be simplified.

(11) Dr. S. D. Zeldin: On the structure of finite continuous groups with a finite number of exceptional infinitesimal transformations.

In a previous paper, presented to the Society in December, 1919, Dr. Zeldin discussed the structure of finite continuous groups having one exceptional infinitesimal transformation. The present paper deals with the structure of groups having any finite number of exceptional transformations.

(12) Mr. H. S. Vandiver: On quadratic congruences and the factorization of integers.

In this paper, Mr. Vandiver considers the problem of the practical determination of the integral values $x$ in the congruence $x^{2} \equiv a(\bmod m)$ where $a$ and $m$ are rational integers prime to each other, $a$ positive. A method of trial and exclusion is given, based on the theory of the quadratic form $u^{2}-a v^{2}$, which supplements another method due to H. J. S. Smith (Collected Papers, vol. 1, p. 148), who used a definite in lieu of an indefinite quadratic form. A similar procedure is convenient in finding the factors of an integer $n$, if $n$ can be expressed in the form $y^{2}-k z^{2}$, where $k$ is a small positive integer.

(13) Professor E. V. Huntington: A mathematical theory of proportional representation.

When $N$ representatives are distributed among the several states, the true quotas are usually fractional, and are necessarily replaced by whole numbers. Various methods of minimizing the injustice involved in these replacements have been proposed (by Willcox, Hill, d'Hondt, etc.), but without satis- 
factory mathematical analysis. Professor Huntington shows that in the case of two states the logarithmic error, $\log (x / y)$, where $x=$ the ratio of the assignments as they actually are, and $y=$ the ratio of the assignments as they ought to be, provides a eomplete criterion for the best apportionment. If there are more than two states, the following principle is proposed: If the injustice belonging to any pair of states can be reduced by a transfer of representatives within that pair, this improvement should be made. It is shown that in any given case there is just one apportionment which is incapable of such improvement, and may therefore be regarded as the best. It is also shown how this best apportionment may be very easily found, by the use of a priority list formed by multiplying the population of each state by the series of factors

$$
\infty, 1 / \sqrt{1 \cdot 2}, 1 / \sqrt{2 \cdot 3}, 1 / \sqrt{3 \cdot 4}, \cdots,
$$

and arranging the results for all the states in order of magnitude.

(14) Dr. H. M. Morse: Recurrent motions of the discontinuous type.

In investigating the nature of the trajectories in a stable dynamical system for unlimited values of the variable which represents the time, Professor Birkhoff has shown that one is led to types of motions which he calls recurrent motions, and which he divides into discontinuous and continuous types. Simple examples of motions of the continuous type can be given. The present paper by Dr. Morse offers the first proof of the existence of recurrent motions of the discontinuous type for the case where the motion is represented by a curve with a continuously turning tangent. This proof is the outcome of a complete classification, from the point of view of analysis situs, of geodesics on surfaces of negative curvature. The paper will be published in the Transactions.

(15) Professor Frank Morley: Presidential Address: Pleasant questions and wonderful effects.

President Morley's address will appear in full in the April number of the BuLLETIN.

(16) Professor Edward Kasner: Properties of orbits in the general theory of relativity.

The orbits in the Einstein theory are the geodesics of the 
four-dimensional manifold $M_{4}$ defined by $d s^{2}=\Sigma g_{i k} d x_{i} d x_{k}$. Professor Kasner considers an arbitrary map of $M_{4}$ upon a 4-flat $(x, y, z, t)$. The properties of the $\infty^{6}$ curves mapping the geodesics are studied. These curves are then projected orthogonally upon a 3 -flat $(x, y, z)$. The $\infty^{1}$ orbits corresponding to a given world-point $(x, y, z, t)$ and a given direction $(d x: d y: d z)$ are shown to have the property that the locus of the centers of curvature is the inverse of a conic. If in particular the $d s^{2}$ is of the static form, studied by Levi-Civita and others, so that the world splits up into ordinary time and space, then it is shown that the conic becomes a circle through the given point; this means that the Meusnier property (relating to the variations of the curvature and the osculating plane) holds. In the Newtonian dynamics of a positional field of force, the locus becomes merely a straight line; since the $\infty^{1}$ trajectories then have a common osculating plane. Some further properties obtained for the general case are too complicated for brief statement.

(17) Professor Edward Kasner: The solar gravitational field in finite form.

In the last of his five notes on the Einstein theory presented at the summer meeting, Professor Kasner showed that there are no four-dimensional manifolds obeying Einstein's equations $G_{i k}=0$ which can be immersed in a 5 -flat; but that for a 6-flat there exist an infinity of solutions, including in particular the solar field. He now examines this field, taken in the Schwarzschild form, in detail. The final result is

where

$$
d s^{2}=-d x^{2}-d y^{2}-d z^{2}+d X^{2}+d Y^{2}-d Z^{2},
$$

$$
\begin{gathered}
X=\sqrt{\frac{r-2 m}{r}} \sin t, \quad Y=\sqrt{\frac{r-2 m}{r}} \cos t, \\
Z=\sqrt{m} \sqrt{\frac{2 r^{3}+m}{r^{3}(r-2 m)}} d r,
\end{gathered}
$$

where $m$ is the mass of the sun, and $r^{2}=x^{2}+y^{2}+z^{2}$. We thus have in finite form a model of the solar field. The model is situated in a flat space of six dimensions; or, using the more exact terminology of Weyl, in an affine-euclidean space of $(2+4)$ dimensions. 
(18) Dr. Norbert Wiener: The average of an analytic functional.

Dr. Wiener discusses in this paper the notion of the average value of a functional which can be expanded in a series of multiple integrals, and develops an average which, over a considerable range of cases, is identical with that treated in his other papers, but which will apply directly to functionals that are not bounded.

(19) Dr. Norbert Wiener: The average of a functional.

Dr. Wiener gives a definition of the average value of a functional which reduces to a special case of the type of Daniell integral already treated by him in his paper entitled The mean of a function of arbitrary elements, presented to this Society at its meeting in December, 1919. The average thus developed is in intimate relation with the theory of probabilities, for it is based on the assumption that the distribution of the values of $F\left(T_{1}+T_{2}\right)$, where $F$ ranges over all continuous functions such that $F\left(T_{1}\right)$ has a certain determinate value $X_{1}$, is what is known statistically as a normal distribution and is independent of $T_{1}$ and $X_{1}$.

(20) Dr. Norbert Wiener: Further properties of the average of a functional.

Dr. Wiener defines the average of the bounded continuous functional $F$ as

$$
\begin{aligned}
& A\{F\}=\lim \pi^{-n / 2} \stackrel{n}{\Pi}\left(x_{k}-x_{k-1}\right)^{-1 / 2} \int_{-\infty}^{+\infty} \cdots \int_{-\infty}^{+\infty} F\{\{\Psi\}(x)\} \\
& \quad \times \exp \left\{-\frac{y_{1}^{2}}{x_{1}^{2}}-\frac{\left(y_{2}-y_{1}\right)^{2}}{x_{2}-x_{1}}-\cdots-\frac{\left(y_{n}-y_{n-1}\right)^{2}}{x_{n}-x_{n-1}}\right\} d y_{1} \cdots d y_{n},
\end{aligned}
$$

where $x_{0}, \cdots, x_{n}$ is a set of numbers satisfying the conditions

$$
x_{0}=0, \quad x_{n}=1, \quad x_{k+1}>x_{k} \text { for every } k,
$$

and where

$$
\{\Psi\}(x) \equiv\left\{\Psi\left(x_{1}, \cdots, x_{n}\right)\left(y_{1}, \cdots, y_{n}\right)\right\}(x)
$$

is that function of $x$ which for $x_{k}<x<x_{k+1}$ assumes the value

$$
y_{k}+\frac{x-x_{k}}{x_{k+1}-x_{k}}\left(y_{k+1}-y_{k}\right) \quad\left(y_{0}=0\right) .
$$


The operation $\lim$ refers to the limit taken as the $x$ 's fill the interval from 0 to 1 more and more closely.

Dr. Wiener shows that $A\{F\}$ exists for every bounded continuous $F$. He shows, moreover, that the notion of average defined here is capable of a Daniell extension which is identical with the notion of average defined in the preceding paper.

(21) Professor Gillie A. Larew: The Hilbert integral and Mayer fields for the problem of Mayer in the calculus of variations.

A. Mayer and Bolza have shown that it is possible to extend to the problem of Lagrange in the calculus of variations the Hilbert theory in which an invariant integral is utilized in the proof that a certain set of conditions is sufficient for the existence of an extremum. A similar extension of this theory to the problem of Mayer is the subject of Professor Larew's paper. An integral is constructed effective in establishing a Weierstrass theorem, and it appears that the imposing of conditions for this integral to be independent of the path is equivalent to placing on the field of extremals conditions analogous to those characterizing the Mayer fields well known to students of the Lagrange problem. The study is confined to a Mayer problem in non-parametric representation and with fixed end-points. It may be noted that Kneser, using other methods, has found results consistent with these for the same problem in parametric form.

(22) Professor R. M. Mathews: Generalizations of the classical construction of the strophoid.

In the classical construction of the strophoid the curve is defined as the locus of the intersection of a pencil of circles with a pencil of lines. The circles have their centers on a line $l$ and all pass through a point $O$ on $l$. Each circle corresponds to that line of the pencil $(F)$ which passes through its center. $O$ is the node, $F$ is the singular focus, and $l$ is parallel to the asymptote. Professor Mathews generalizes this construction in two ways: first, for a pencil of circles through the node with their centers on any line through that point; and second, for a pencil of circles through any two points whose tangents meet on the curve.

(23) Professor W. A. Hurwitz: Some properties of methods of evaluation of divergent sequences. 
In case a linear transformation, $y_{n}=\sum_{k=1}^{n} a_{n k} x_{k}$, carries every convergent sequence $\left(x_{n}\right)$ into a sequence $\left(y_{n}\right)$ converging to the same value as $\left(x_{n}\right)$, the transformation is said to be regular; necessary and sufficient conditions for regularity are furnished by the Silverman-Toeplitz theorem. In the present paper, Professor Hurwitz investigates the conditions under which a regular transformation of real elements maintains the property of regularity even in the case of the improper limits $\pm \infty$, and also the conditions under which it carries a divergent sequence into another which, if it is not convergent, at least has its limits of oscillation not further separated than in the original sequence. Application is made to the particular case of analytically regular transformations, as defined by Hurwitz and Silverman.

(24) Professor W. C. Graustein: Parallel maps of surfaces.

Two real surfaces, in continuous one-to-one point correspondence, with the directed normals parallel at corresponding points, are said to correspond by a parallel map; or more specifically, by a directly parallel or an inversely parallel map, according as corresponding directions of rotation about corresponding points are the same or opposite. In Professor Graustein s paper, parallel maps are further classified as hyperbolic, elliptic, or parabolic, after the manner of the classification of one-dimensional projective correspondences, and each parallel map is characterized by an invariant analogous to the invariant of such a correspondence. The classification of parallel maps thus obtained is instructive in the light it sheds on known theorems concerning parallel maps, and it proves to be of value in developing new results.

(25) Professor J. H. M. Wedderburn: On the maximum value of a determinant.

Professor Wedderburn gives a proof, due to J. Schur (Mathematische AnNalen, vol. 66 (1909), pp. 488-510), of Hadamard's theorem that the maximum value of the determinant of a matrix $A=\left(a_{r s}\right)$ is $|A| \leqq \Sigma\left(\left|a_{r s}{ }^{2}\right| / n\right)^{n / 2}$. As this inequality was not the main purpose of Schur's paper, his exposition does not show how brief the proof really is. If $\bar{a}_{r s}$ denotes the conjugate imaginary of $a_{r s}$ and $\vec{A}=\left(\bar{a}_{r s}\right)$, then $\bar{A}^{\prime} A$ is a positive Hermitian form whose roots, as is well known, are real and positive. The sum of these roots is $\Sigma_{r s} a_{r s} \bar{a}_{r s}=\Sigma\left|a_{r s}{ }^{2}\right|$; 
hence their product is less than or equal to $\left(\Sigma\left|a_{r s}{ }^{2}\right| / n\right)^{n}$. This product is equal to the determinant of $A^{\prime} \bar{A}$ which is equal to the absolute value of $|A|^{2}$, thus proving the required inequality.

(26) Professor J. H. M. Wedderburn: On the automorphic transformation of a bilinear form.

The problem of the automorphic transformation of a bilinear form is usually discussed on the basis of the Cayley-Hermite solution. This solution is rational but becomes indeterminate under certain conditions. Professor Wedderburn derives a very simple solution in transcendental form which includes the exceptional cases and reveals their analytical nature clearly.

(27) Professor J. W. Lasley, Jr.: Some special cases of the flecnode transformation of ruled surfaces.

The process of obtaining the flecnode surface of a ruled surface leads to the consideration of a suite of surfaces, called the flecnode suite. Questions arise as to the cases in which this suite terminates or returns into itself. It is the purpose of Professor Lasley's paper to study some of these questions. It is found that the suite terminates with its first transform if and only if the given ruled surface has a straight line directrix. In case the termination occurs with the second transform, both branches of the flecnode curve on the given ruled surface may be obtained without integration. The suite is of period two if and only if the flecnode curve meets every generator in two coincident points. The flecnode suite cannot be of period three, nor of period four. The conditions for termination and periodicity considered are expressed in terms of the invariants of the differential equations which define the given ruled surface.

(28) Professor R. G. D. Richardson: The theory of relative maxima and minima of quadratic and hermitian forms and its application to a new foundation for the theory of bilinear forms. First paper: Equivalence of pairs of bilinear forms.

The theory of maxima and minima, which plays an ever increasing rôle in analysis, suggests a simpler and direct method for the discussion of a pair of forms. In the case of quadratic or hermitian forms, one of which is definite, each zero of the $\lambda$-determinant can be interpreted as a maximum 
of a certain isoperimetric problem and as a minimum of another. This point of view enables Professor Richardson in this simplest case to obtain a direct method for reducing the pair of forms to the normal type as follows. Denoting by $\lambda_{1}, \cdots, \lambda_{n}$ the zeros of the $\lambda$-determinant, and setting $\lambda=\lambda_{1}$ in the corresponding $\lambda$-matrix ||$a_{i j}-\lambda b_{i j}||$, the rows are regarded as the coefficients of linear homogeneous equations and the solution used as the first row of a matrix. The other rows are obtained in the same way by setting in the matrix $\lambda=\lambda_{2}, \cdots, \lambda_{n}$. The elements of the columns also serve as coefficients of linear equations which determine a second matrix. In the case of quadratic forms, this is identical with the first. When the $\lambda$-matrix is multiplied by these two matrices it is reduced to one with terms in the main diagonal only.

By a generalization of this process suggested by the formal work in the problem of taking the relative extreme, any pair of non-singular bilinear forms is reduced to its normal type. When the multiplicity of any $\lambda_{i}$ is greater than its index (the number of linearly independent solutions of the corresponding linear equations) some of the rows and columns of the multiplying matrices are solutions of non-homogeneous equations extended from the original homogeneous equations by a process resembling the taking of a derivative.

The equivalence of pairs of bilinear forms is then based on three simple notions: (1) the equivalence of polynomials in $\lambda$; (2) the equality of the indexes of $\lambda_{i}$; (3) the possibility of solution of certain non-homogeneous linear equations.

(29) Dr. J. S. Taylor: The analytic geometry of complex variables with some applications to function theory.

Dr. Taylor's paper lays the foundations for a development of the analytic geometry of complex variables and applies some of the results to a geometric interpretation of function theory. A pair of complex numbers $X$ and $Y$ is represented by a point in four-space. Several definitions of distance are discussed, a geometric meaning is given to a complex angle, and six new pseudo-trigonometric functions of a complex angle are defined. The surfaces representing equations of the first and second degree in two complex variables are investigated in detail. Finally, a comparatively simple geometric meaning is given to the derivative of a function of a single complex variable, to its differential, and to its integral. 
(30) Professor C. H. Forsyth: The value of a bond to be redeemed ultimately, both principal and interest, in equal installments.

The formulas for computing the value of a bond to yield a specified rate of interest have been derived for all cases where the principal is to be redeemed in one sum or in equal installments. The formula has been derived for the case where both principal and dividend are to be redeemed in equal annual installments. Professor Forsyth derives the formula for the more general case where the principal and dividend are to be redeemed in equal installments, but where the first installment is to be repaid at the end of $f$ years and the rest at intervals of $t$ years.

(31) Professor C. H. Forsyth: Valuation of bonds bought to realize a specified rate of interest, assuming the amortizations to accumulate at a savings-bank rate.

Investors in bonds realize only too well the usual impossibility of obtaining the rate of interest corresponding to the price computed by the ordinary formulas simply because the amortizations are relatively small sums, and small sums can rarely demand as high rates of interest as can such large sums as the principals of the bonds. Professor Forsyth derives a formula for computing the price of the conventional bond to yield actually a specified rate of interest since the amortizations are assumed to accumulate at a practical rate of interest, such as that of a savings bank. The formula thus derived proves singularly convenient for the solution of the inverse problem, which is the determination of the rate of interest that a given price will yield. In fact, the solution of the inverse problem is almost as easy and quite as simple as that of the direct problem.

(32) Dr. Einar Hille: Zeros of Legendre functions.

In this paper Dr. Hille considers solutions of Legendre's differential equation

$$
\left(1-z^{2}\right) \frac{d^{2} w}{d z^{2}}-2 z \frac{d w}{d z}+a(a+1) w=0
$$

with respect to the location of their zeros in the complex plane. The distribution of zeros along rays from $z=+1$ is investigated by means of two fundamental integral equalities which 
are generalizations of well known formulas generally attributed to Green. It is proved that $P_{a}(z)$, the solution regular at $z=+1$, has its zeros in the interval $(-1,+1)$ when $a$ is real, and in the interval $(+1,+\infty)$ when $a=-\frac{1}{2}+\mu i$. The number of zeros has been previously determined by Dr. Hille. When $a=\lambda+\mu i\left(\lambda>-\frac{1}{2}, \mu>0\right)$ it is proved that the zeros lie in a certain sector of the upper half of the plane. Finally, the solution $Q_{a}(z)$ which belongs to the exponent $1+a$ at infinity is shown to have no zeros in the finite plane as long as $\lambda>-\frac{1}{2}$.

(33) Professor W. B. Carver: Systems of linear inequalities.

In a paper on Systems of linear inequalities (ANNALS of Mathematics, vol. 20, p. 191, March, 1919), Professor L. L. Dines gives a necessary and sufficient condition for the existence of solutions of such a system. His conditions are expressed in terms of the $I$-rank of the matrix, the definition of $I$-rank being somewhat analogous to that of rank.

In the present paper Professor Carver gives a necessary and sufficient condition that solutions of the system should not exist, the condition being expressed in a form similar to that ordinarily used to define linear dependence. Independence of a system and equivalence of two systems are then defined, and necessary and sufficient conditions found for them.

(34) Professor J. L. Coolidge: Differential geometry of the complex plane.

Professor Coolidge's paper is devoted to the study of systems of points in the complex plane, whose coordinates are analytic functions of two real variables, yet which do not lie on a real curve. Some attention is also paid to three-parameter systems.

(35) Professor C. L. E. Moore: Note on minimal varieties in hyperspace.

Professor Moore's paper appeared in the February number of this Bulletin.

(36) Professor I. J. Schwatt: Independent expressions for the Bernoulli numbers.

Laplace, Stern, Kronecker, Shovelton and others have developed independent expressions for the Bernoulli numbers. Professor Schwatt has established methods which have 
enabled him to find new independent expressions for these numbers. The methods are believed to be more direct and the results more simple than those obtained heretofore.

(37) Professor I. J. Schwatt: Relations involving the numbers of Bernoulli and Euler.

By the methods of the preceding paper, Professor Schwatt has developed new relations involving the numbers of Bernoulli and Euler.

(38) Professor I. J. Schwatt: Independent expressions for Euler numbers.

Very few independent expressions for the Euler numbers have been developed. The simplest among them is the one by Worpitzky (Journal Für Mathematik, vol. 94, p. 203). The expressions by Scherk (ibid., vol. 4, p. 299), and by Saalschütz (Vorlesungen über die Bernoullischen Zahlen, p. 100) are rather complicated. By means of operations with series Professor Schwatt has been enabled to devise methods and to obtain results which are believed to be quite simple.

(39) Professor I. J. Schwatt: Independent expressions for the Euler numbers of higher order.

Lucas (Bulletin DE LA Société Mathématique DE France, vol. 6, p. 53) has introduced the Euler number of higher order. Professor Schwatt has been able to find only two independent expressions representing these numbers, one by Ely (American Journal of Mathematics, vol. 5, p. 339) and the other by Shovelton (Quarterly Journal of MatheMATICs, vol. 46, p. 220). By means of operations with series methods have been devised which lead to simple independent expressions of these numbers. Three independent expressions for $T_{n p}$, the coefficient of $x^{2 n+p} /(2 n+p)$ ! in the expansion of $\tan ^{p} x$, are also given.

(40) Professor I. J. Schwatt: Summation of a type of Fourier's series.

The principles used in the direct summation of

$$
\sum_{n=0}^{\infty}(-1)^{n} \frac{\sin (a+n g)}{b+n h} r^{n},
$$

when $r$ may be 1 , in which case $g \equiv 0(\bmod 2 \pi)$, is first applied to $\sum_{n=0}^{\infty}(-1)^{n}[1 /(b+n h)]$. The method given by Professor 
Schwatt in this paper is more general in its application than the one which he has presented in an article in the Nouvelles Annales de Mathématiques, (4), vol. 6, pp. 203-209 (May, 1916).

(41) Professor F. W. Owens: On the projectivity assumption in projective geometry.

Various forms of the projectivity assumption in projective geometry have been made by different writers. A number of these are given and shown to be equivalent in Veblen and Young's Projective Geometry. In this paper, Professor Owens derives the ordinary forms of statement from milder forms of the assumption.

(42) Professor R. W. Burgess: On certain simple skew frequency curves.

To facilitate the discussion of frequency distributions which do not conform to the normal probability curve, Pearson in 1895 obtained a series of skew frequency curves by integrating the differential equation

$$
\frac{1}{y} \frac{d y}{d x}=\frac{a+b x}{c+d x+e x^{2}} .
$$

This equation is a generalization of the slope property of the normal curve

$$
\frac{1}{y} \frac{d y}{d x}=-a x .
$$

The aim of the present paper is to give, by considerations based on the elementary theory of probability, a new basis for some of Pearson's curves, and to propose modifications in his methods of fitting these curves to given data. Professor Burgess' point of view is that the individual items summarized by the frequency curve may be regarded as being deviations all in one direction from a fixed origin, and, in the discrete case, as being built up by successive increments, the probability of adding each increment being variable. Among the curves derived on this basis are those of the family $y=c x^{n} e^{-k x}$. These curves are one type of those introduced by Pearson, and are also cases of Knibbs' flexible curve.

In view of the fundamental importance of the area in work with frequency curves, Professor Burgess proposes to use, as the criterion for goodness of fit, the sum of the differences of 
area from the origin to various values of $x$, between the actual frequency distribution and the trial curve. The trial curves for this method are obtained by the use of a table giving the percentage of area to the right of the centroid for important values of $n$. This method requires the construction of tables of areas of these curves, that is, of the integral

$$
I(x, n)=\int_{0}^{x} x^{n-1} e^{-x} d x / \Gamma(n) .
$$

Such tables may be easily constructed for values of $n$ which are integral multiples of one-half by the use of the difference equation

$$
I(x, n+1)-I(x, n)=-y(x, n+1)
$$

and of tables of the exponential and the error functions.

(43) Dr. G. M. Robison: Divergent double series and sequences.

For any given double sequence $s_{m n}$, a new double sequence $\sigma_{m n}$ may be defined by a linear transformation:

$$
\sigma_{m n}=\sum_{k=1, l=1}^{m, n} a_{m n k l} \cdot s_{k l} .
$$

Dr. Robison establishes a necessary and sufficient condition that the transformation be regular, i.e., that when it is applied to a bounded convergent sequence the new sequence shall be convergent to the same value as the original sequence. Further investigations determine when a regular transformation carries a sequence which becomes positively infinite into a sequence which becomes positively infinite. The condition that a transformation transform a bounded convergent sequence $\left(s_{m n}\right)$ into a bounded convergent sequence $\left(\sigma_{m n}\right)$, the limits not necessarily being the same, is given; also the condition that a transformation carries a bounded sequence into a bounded convergent sequence. These theorems are also extended to the case where the new sequence is defined as follows:

$$
\sigma_{m n}=\sum_{k=1, l=1}^{\infty, \infty} a_{m n k l} \cdot s_{k l},
$$

each element $\sigma_{m n}$ depending upon all the elements of the original sequence. 
(44) Professor G. D. Birkhoff: An extension of Poincarê's geometric theorem.

The hypothesis of Poincaré's geometric theorem* requires that the two boundaries of a ring be invariant. As Professor Birkhoff shows, it is sufficient to require the invariance of only one of these boundaries. The extension is very convenient for the dynamical applications.

(45) Dr. J. L. Walsh: On the location of the roots of polynomials.

Dr. Walsh proves that if the points $a_{1}, a_{2}, \cdots, a_{k}$ lie on or within a circle whose center is $\alpha$ and radius $r_{1}$ and if the points $b_{1}, b_{2}, \cdots, b_{k}$ lie on or within a circle whose center is $\beta$ and radius $r_{2}$, then all the roots of the polynomial

$$
\begin{aligned}
f(z)=\left(z-a_{1}\right) & \left(z-a_{2}\right) \cdots\left(z-a_{k}\right) \\
& -A\left(z-b_{1}\right)\left(z-b_{2}\right) \cdots\left(z-b_{k}\right), \quad A \neq 1,
\end{aligned}
$$

lie on or within the $k$ circles whose common radius is

$$
\left(r_{1}+\left|A^{1 / k}\right| r_{2}\right) /\left(1+\left|A^{1 / k}\right|\right)
$$

and whose centers are the $k$ points $\left(\alpha-\beta A^{1 / k}\right) /\left(1-A^{1 / k}\right)$, where $A^{1 / k}$ takes all the $k$ values possible. If any one of these $k$ circles is exterior to all the others, that circle contains precisely one root of $f(z)$. This theorem holds with a very slight change if $A=1$. There is a similar theorem for the polynomial $\left(z-a_{1}\right)\left(z-a_{2}\right) \cdots\left(z-a_{k}\right)-A=0$.

Miss Mullikin was introduced by Professor R. L. Moore, and Dr. Hille by Professor G. D. Birkhoff. In the absence of the authors, the papers of Professor Wilder, Dr. Walsh, Miss Mullikin, Dr. Schweitzer, Dr. Rowe, Mr. Vandiver, Professor Lasley, Professor Moore, and Professor Birkhoff were read by title, and Professor Schwatt's papers were read by Professor H. H. Mitchell. Dr. Zeldin's second paper, Professor Kasner's first, Dr. Wiener's second, and Professor Forsyth's first paper were also read by title.

R. G. D. Richardson,

Secretary.

* An interesting attempt at a direct demonstration of the theorem has been given recently by Mr. E. Gau, Bulletin des Sciences MathémaTIQUES vol. 43 (1919), p. 12-17. Unfortunately it is tacitly assumed (p. 14) that radial lines are carried over into curves met by any radial line only once. 